\title{
Immune thrombocytopenia in patient with clear cell renal carcinoma: case report and literature review
}

\begin{abstract}
Paraneoplastic secondary immune thrombocytopenia is a rare disorder; however, this entity may delay the timely treatment of the underlying cancer due to the limiting side effects of therapy. There are few reported cases of immune thrombocytopenia associated with clear cell renal carcinoma and it is described that they may have a good response to glucocorticoids and immunoglobulin.

Case report: We present here the case of a 70-year-old patient with a diagnosis of clear cell renal carcinoma with associated severe thrombocytopenia that was initially diagnosed as disseminated intravascular coagulation lasting several months without recovering the platelet count and therefore the patient could not initiate anticancer therapy timely. Subsequently, the diagnosis was redefined to secondary immune thrombocytopenia after a detailed analysis of the blood count and to achieve a good response with the use of oral prednisone. The objective of this report is to describe the association of some solid neoplasms with immune thrombocytopenia, the potential benefit of systemic glucocorticoid therapy, which may allow timely directed therapy for these patients.
\end{abstract}

Keywords: clear cell metastatic renal cell carcinoma, autoimmune thrombocytopenia
Volume 4 Issue I - 2020

\author{
Mart nez-Cordero Humberto, Salgado-Reyes \\ Paula, Alcala-Lara Sebastian, Linares-Pati o, \\ Maximo Mejia,Angie Ramos Robert \\ Hematology and Bone Marrow Transplantation, Instituto \\ Nacional de Cancerolog a Universidad El Bosque, Colombia
}

\begin{abstract}
Correspondence: Martínez-Cordero Humberto, Instituto Nacional de Cancerologia, Hematology and Bone Marrow Transplantation Salgado-Reyes, Paula, Universidad El Bosque, Colombia,Tel 31566944I9, Email humbertomartinez4@hotmail.com
\end{abstract}

Received: January 16, 2020 | Published: February 07, 2020

\section{Introduction}

Clear cell renal carcinoma corresponds to $80-85 \%$ of all primary renal neoplasms, with an incidence, mortality and prevalence of 5.3, 2.3 and 13.4 per 100,000 inhabitants respectively. ${ }^{1,2}$ The incidence varies by region, as being with the Czech Republic and North America being the areas with the highest incidence and prevalence with around 30.5 and 18.5 per 100,000 inhabitants, while in Colombia there is a figure of 3.6 per 100,000 inhabitants. It happens more in the male than female population, with a ratio of $3: 1^{3}$ and occurs mostly in people in the sixth and eighth decade of life. ${ }^{1,2}$ The five-year survival rate has doubled in recent decades. This tendency is due to early detection when the primary tumor is in the early stages $(<4 \mathrm{~cm}) .{ }^{1,2}$ The recommended first-line treatment in the advanced stages is tyrosine kinase inhibitors (Sunitinib and Pazopanib) until disease progression or increased toxicity. ${ }^{4,5}$ The use of these medications requires a platelet count between 75,000-50,000 cells per microliter and the management should be postponed until the recovery of this cell line due to an increased risk of bleeding, however, it is to be recognized that clear cell renal carcinoma could be associated with paraneoplastic phenomena including immune thrombocytopenia which correspond to the production of antibodies that cause platelet destruction. ${ }^{6,7}$

We present here a case of a patient with clear cell renal carcinoma that was initially approached as disseminated intravascular coagulation, however, given the characteristics of the blood count, peripheral blood spread and the good response to the use of systemic corticosteroids, it was determined that he was actually suffering from paraneoplastic secondary immune thrombocytopenia. The objective of this case report is to show the association of solid cancers, such as a clear cell renal carcinoma, with immune thrombocytopenia and the benefit of the use of systemic steroids that allow the timely initiation of targeted therapy that leads to an improvement in the survival.

\section{Case presentation}

A 70-year-old man, a native of San Gil - Santander Colombia and living in Bogotá, who was treated for the first time in the oncology department of the National Cancer Institute of Colombia in May 2015 with a diagnosis of advanced clear cell renal carcinoma T4NXM1 (lung and testicular metastasis) not a candidate for surgery but a tyrosine kinase inhibitor requirement for disease control. Thrombocytopenia was found since admission, so the concept of hematology was requested to start sunitinib. At assessment, mild microcytic and hypochromic anemia and severe thrombocytopenia were found. A complete bone marrow study is requested, which was normal. A low level of vitamin B 12 and ferritin was documented, so it was replaced waiting for recovery of the platelet count. The response was null requiring continuous platelet transfusion without allowing the onset of tyrosine kinase inhibitor.

An initial diagnosis of disseminated intravascular coagulation associated with solid neoplasia was made. It was considered that it required treatment of the primary neoplasm for the improvement of platelet count, however it was considered that there were some characteristics that suggested associated autoimmune component such as normality in other cell lines, large platelet size and absence of bleeding with very low counts. It was then decided to start prednisone at $0.5 \mathrm{mg}$ per kilogram, achieving a recovery in platelet count at more than 100,000 cells per microliter, which allowed starting treatment with pazopanib in March 2017. During the course of treatment, thrombosis of the inferior vena cava was documented that required full anticoagulation. The following graph describes the complete trend of platelet count evolution. Subsequently, in September 2018, he consulted the emergency department for abdominal pain and emetic syndrome. Acute renal failure was documented on the status of chronic kidney disease with emergency dialysis criteria, however, 
it was considered that the clinical status with respect to metastatic pathology did not benefit from renal replacement therapy and also differs from resuscitation maneuvers, a wish also accepted by family members. Finally, in October 2018, he died because of his underlying illness.

\section{Discussion}

Immune thrombocytopenia secondary to renal carcinoma is a rare entity. Its incidence and prevalence are 1 to 5 cases and 8 cases per 100,000 inhabitants respectively, both increase with age and sex (higher in men). ${ }^{67}$ Patients presenting with this paraneoplastic syndrome may debut with an asymptomatic condition (in most cases) or the presence of bleeding, petechiae, purpura, epistaxis and/or fatigue. Normally, immune thrombocytopenia is a diagnosis of exclusion in which there should be a platelet count of less than $100,000 / \mathrm{uL}$ without being related to anemia or leukopenia, ${ }^{8}$ and other causes should be ruled out, so other diagnostic tests may be requested that help the exclusion of other entities, which usually include the complete study of bone marrow in certain cases. ${ }^{9,10}$

If immune thrombocytopenia is secondary to a solid tumor, the first step is to treat the neoplasm in order to correct the defect, $4,6,10,11$ however, in cases such as the one proposed in this review, where treatment Surgical was not possible, the proposed first-line therapy is the use of glucocorticoids and/or intravenous immunoglobulin. The use of intravenous immunoglobulin generally presents a faster response, however, the use of this therapy is only indicated in patients with active bleeding or who require an invasive emergency intervention. ${ }^{6,10}$ A comparative study was developed on the use of glucocorticoids versus the use of intravenous immunoglobulin where both treatments were shown to have a similar response when supplemented with oral prednisone for three weeks, however, it should be noted that the use of oral prednisone or dexamethasone in high doses for one or two weeks with a subsequent gradual decrease shows the same efficacy as the therapy mentioned above. ${ }^{6}$ In addition to the above, the use of splenectomy, rituximab or immunosuppressive therapy has also been proposed as a second line of treatment, taking into account that these treatments should only be considered in case the first line does not demonstrate an adequate response and platelet count persists below $20,000 / \mathrm{uL}^{10}$

\section{Conclusion}

It is very important, not only in the case of renal carcinoma, but in general in all malignant pathologies, to make an early diagnosis to offer a curative therapy. In addition, it is important to consider all the paraneoplastic phenomena or syndromes that may occur during the course of the disease to reduce complications and offer timely treatment. In the specific case of immune thrombocytopenia secondary to clear cell renal carcinoma, rapid onset of glucocorticoids gives an important benefit and, as demonstrated in the proposed case, a complete resolution of thrombocytopenia can be achieved, thus reducing readmissions hospital, due to transfusion requirements and/ or due to complications associated with the presentation of decreased platelet values, in addition to being able to provide the recommended therapy according to oncological guidelines.

\section{Thanks}

We appreciate the collaboration with the Pathology Department of the National Cancer Institute for their great work in the study of patients and the contribution provided for this study.

\section{Note of reservation}

The National Cancer Institute is not responsible for the concepts issued by researchers in their work, it will only ensure scientific rigor. The same methodological and ethical for the search of truth and justice.

\section{Acknowledgments}

None.

\section{Conflicts of interest}

The author declares there is no conflict of interest.

\section{References}

1. Ariana Znaor, Joannie Lortet-Tieulentb, Mathieu Laversannea, et al. International Variations and Trends in Renal Cell Carcinoma Incidence and Mortality. European Urology. 2015;67(3):519-530.

2. J Ferlay, E Steliarova-Fouchera, J Lortet-Tieulenta, et al. Cancer incidence and mortality patterns in Europe: Estimates for 40 countries in 2012. European Journal of Cancer. 2013;49(6):1374-1403.

3. World Health Organization. International Agency for Research on Cancer. 2018.

4. Instituto Nacional del Cancer. Tratamiento del cáncer de células renales - Versión para profesionales de salud. 2019.

5. Cancer Insititute N. Renal cell metastatic sunitinib. 2019.

6. Anam Rehan, Aakash Desai, Corina Iorgoveanu, et al. Secondary Immune Thrombocytopenic Purpura in Renal Cell Cancer: A Paraneoplastic Syndrome. Cureus. 2019;11(4):e4398.

7. Shigekatsu Maekawa, Masayoshi Nagata, Hiroshi Watanabe, et al Complete Response of severe idiopathic thrombocytopenic purpura after resection of bulky chromophobe renal cell carcinoma. Cancer Cell \& Microenvironment. 2016;3:e1158.

8. Kevin R Melnick, Courtney Chang, Ana Antun, et al. Paraneoplastic Thrombocytopenia Cured With Nephrectomy and Vena Cava Thrombectomy: Concurrent Hematology and Oncology Management Conundrums. J Oncol Pract. 2017;13(11):767-768.

9. Cindy Neunert, Wendy Lim, Mark Crowther, et al. The American Society of Hematology 2011 evidence-based practice guideline for immune thrombocytopenia. Blood Journal. 2011;17(16):4190-4207.

10. American Society of Hematology. ASH Pocket Guides. 2011.

11. Eun Ju Cho, Ha Jung Lee, Kyung Un Park, et al. Immune thrombocytopenic purpura in a patient with renal cell carcinoma. Korean J Intern Med. 201429(5):671-674.

12. American Society of Hematology. Don't treat patients with immune thrombocytopenic purpura (ITP) in the absence of bleeding or a very low platelet count. 2014. 